American Journal of Applied Sciences 7 (10): 1317-1320, 2010

ISSN 1546-9239

(C) 2010 Science Publications

\title{
Quality evaluation of Ketoprofen Solid Dosage Forms Available in the Pharma-Market of Bangladesh
}

\author{
${ }^{1}$ Kamrun Naher, ${ }^{1}$ Gazi Md. Monjur Murshid, ${ }^{1}$ Md. Golam Hossain, \\ ${ }^{1}$ Md. Amirul Islam, ${ }^{2}$ Sheik Sayeed Hasan and ${ }^{3}$ Sarder Nasir Uddin \\ ${ }^{1}$ Pharmacy Discipline, Life Science School, Khulna University, Khulna, 9208, Bangladesh \\ ${ }^{2}$ Department of Pharmaceutical Technology, University of Dhaka, Dhaka, 1000, Bangladesh \\ ${ }^{3}$ Biotechnology and Genetic Engineering Discipline, Life Science School, \\ Khulna University, Khulna, 9208, Bangladesh
}

\begin{abstract}
Problem statement: Ketoprofen, a widely used analgesic and anti-inflammatory drug, was available in two types of solid dosage forms in the pharma-market of Bangladesh: enteric-coated tablet and capsule of sustained-release pattern. Seven brands of ketoprofen enteric-coated tablets and four brands of ketoprofen sustained release capsules were studied for their in-vitro release behavior as well as potency status. Approach: The studies were carried out to compare with standard sample of ketoprofen sustained-release pellets and that of ketoprofen powder. To determine the release pattern of the preparations, disintegration study and dissolution tests were performed as per the method described in standard pharmacopeial compendia (British Pharmacopoeia or BP and United State Pharmacopoeia or USP respectively). The potency of the samples were determined by the UV spectroscopic method as described in BP. Results: Out of seven samples of tablets, two brands (KT-03 and KT-07) were found noncompliant in respect of disintegration test in acid stage whereas rest of the brands complied with BP specification in buffer stage at $\mathrm{pH}$ 6.8. The dissolution study of ketoprofen tablets were carried out in both acid and buffer stages and all the samples satisfied with USP specification in both stages. All of the brands of ketoprofen capsule also complied with the USP specification. Two brands (KT-03 and KT-07) of tablets were found non-compliant whereas rest brands of tablets and all brands of capsules exerted compliance in respect of potency. Conclusion: This study will provide a basis for further invivo bioavailability studies of these brands to draw a more conclusive remark regarding quality status of these samples.
\end{abstract}

Key words: Ketoprofen, enteric-coated tablet, sustained-release, disintegration test, dissolution test, solid dosage, compendia, pharmacopoeia, spectroscopic method, potency estimation

\section{INTRODCTION}

Ketoprofen, chemically 2-(4-isobutylphenyl) propionic acid, is a nonsteroidal anti-inflammatory and analgesic drug used widely in the treatment of patients with rheumatic diseases. It acts by inhibiting Cyclooxygenase-1 (COX-1) and cyclooxygenase-2 (COX-2) activities and thereby inhibiting the Synthesis of prostaglandin (Parker, 2005). The prevalence of rheumatic diseases is increasing day by day in our country, especially among elderly and that creates a demand of quality analgesic drugs. The aim of the study is to investigate the release pattern and potency status of different preparations of Ketoprofen solid dosage forms so that quality brand (s) could be identified.
The valid reasons for coating tablets include: (i) To control the site of release of drug which is best illustrated in terms of enteric coating. (ii) to provide a controlled, continuous drug release rate. Now-a-days, a lot of tablet preparations are coated to make a product safe, elegant, stable, therapeutically effective and aesthetically nice. After administration, the coating of the tablets should be ruptured quickly and active ingredient is to be released from dosage form for rapid absorption. The release pattern of a coated tablet can be revealed by disintegration and dissolution tests (Chambliss et al., 1984).

Sustained release dosage form of a drug contributes some advantages. Since the frequency of drug administration is reduced, patient compliance is improved and drug administration can be made more

Corresponding Author: Sarder Nasir Uddin, Biotechnology and Genetic Engineering Discipline, Khulna University, Khulna, 9208, Bangladesh Fax: 88-041-731244 
convenient as well. Blood level fluctuation characteristic of multiple dosing of convenient dosage form is reduced, because a more even blood level is achieved through sustained release dosage form (Lachman et al., 1986).

A large number of studies have been conducted previously on the dissolution and disintegration pattern of ketoprofen dosage forms: (patents.nemblewisdom.com, Pharm. Res., 1994, Microencapsule, 1992, www.ualberta.ca/JPPS/colon.htm). All these extensive studies were conducted to evaluate mainly the in-vivo bioavailability pattern of ketoprofen formulations. The main objective of the present study was to focus the release pattern and potency status of the ketoprofen solid dosage forms available in the local market of Bangladesh, so that quality brands in the local market could be identified. This research team successfully conducted study on Indomethacin, a member of the same therapeutic group to identify the quality status of the generic earlier (Murshid et al., 2006).

\section{MATERIALS AND METHODS}

Sample: Seven brands of ketoprofen enteric-coated tablet and four brands of ketoprofen capsule were purchased from the different regions of Bangladesh. The areas include different wholesale and retail medicine shops of Khulna City including Heraj Market (Khulna), Moilapota and Gollamari (Khulna), Savar and Mitford retail market of Dhaka City. The samples were collected in such a way that some samples were immediately after manufacturing, some were just before their expiry dates and others were intermediate of two kinds to know about the stability status of the drug products during their shelf-life. The labels of ketoprofen tablets and ketoprofen capsules claimed to contain $50 \mathrm{mg}$ of ketoprofen per tablet and $100 \mathrm{mg}$ of ketoprofen per capsule respectively. Identifiable codes were given against the brands: KT-01 to KT-07 for tablet brands and $\mathrm{KC}-01$ to $\mathrm{KC}-04$ for capsule brands. This study was carried out at the Biopharmaceutics laboratory of the Pharmacy Discipline of Khulna University.

Chemicals: Standard sample of ketoprofen sustainedrelease pellets and that of ketoprofen powder were collected from Beximco Pharmaceuticals Ltd. and Eskayef Bangladesh Limited respectively to use as reference standard. Disodium hydrogen phosphate, potassium dihydrogen phosphate and trisodium phosphate dehydrate were purchased from E. MERK (India) Ltd.
Disintegration studies: Disintegration test was carried out by Thermonic Tablet Disintegration Test Unit, Campbell Electronics, Mumbai, India. BP specification for disintegration time test: no tablet should show the sign of cracks that would allow the escape of content in acid stage and all tablets must be disintegrated within $60 \mathrm{~min}$ in buffer stage.

Dissolution studies: In-vitro drug release studies were conducted using type II dissolution apparatus at $37 \pm 0.5^{\circ} \mathrm{C}$ in two stages. For calculating the amount of drug dissolved, the calibration curves for both acid and buffer stages were prepared from standard solutions of different concentrations of the respective drug. According to USP specification for dissolution study, drug must be dissolved $<10 \%$ of the labeled amount after $2 \mathrm{~h}$ in acid stage and drug must be dissolved $>80 \%$ of the labeled amount after $60 \mathrm{~min}$ in the buffer stage.

Potency studies: Though titrimetric method has recently been used to determine the potency of ketoprofen bulk (Maheswari et al., 2010) potency of ketoprofen solid dosage form is reported to be estimated accurately by spectrophotometry (Blanco et al., 1997; Aboul-Enein et al., 2003). A UV spectrophotometer (Camspec, UK) was used to determine the amount of ketoprofen present in the samples at $258 \mathrm{~nm}$ and was compared with the standard sample of that drug. BP specification for potency study: $(100 \pm 5) \%$ for tablet preparations and $(100 \pm 10) \%$ for capsule dosage forms.

The experiments were conducted in the Biopharmaceutics and Pharmacokinetics laboratory of Pharmacy Discipline, Khulna University, Khulna-9208, Bangladesh.

\section{RESULTS}

Disintegration test: The results of disintegration test of 7 samples of ketoprofen tablets were shown in Table 1 and Fig. 1 for acid stage and buffer stage respectively.

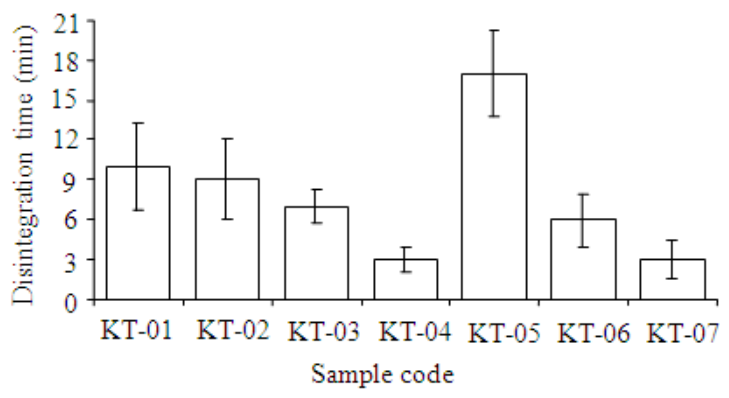

Fig. 1: Disintegration pattern of ketoprofen tablet brands, in buffer stage 
Am. J. Applied Sci., 7 (10): 1317-1320, 2010

Table 1: Disintegration test of ketoprofen tablets, in acid stage

\begin{tabular}{llll}
\hline Sample code & Number of tablets & Status of tablets after $2 \mathrm{~h}$ (in $0.1 \mathrm{M} \mathrm{HCl} \mathrm{media)}$ & Observations \\
\hline KT-01 & 6 & Intact, no cracking & Compliance \\
KT-02 & 6 & Intact, no cracking & Compliance \\
KT-03 & 6 & Small crack found, ingredient may leak. & Non compliance \\
KT-04 & 6 & Intact, no cracking & Compliance \\
KT-05 & 6 & Intact, no cracking & Compliance \\
KT-06 & 6 & Intact, no cracking & Compliance \\
KT-07 & 6 & Small crack found, ingredient may leak. & Non compliance \\
\hline
\end{tabular}

Table 2: Dissolution study of ketoprofen tablet, KT 01 to KT 07 , in acid stage

\begin{tabular}{llll}
\hline Sample code & Number of tablets & Percent drug dissolved after $2 \mathrm{~h}$ in acid stage & Observations \\
\hline KT-01 & 6 & 6.44 & Compliance \\
KT-02 & 6 & 2.09 & Compliance \\
KT-03 & 6 & 1.73 & Compliance \\
KT-04 & 6 & 5.40 & Compliance \\
KT-05 & 6 & 4.38 & Compliance \\
KT-06 & 6 & 6.84 & Compliance \\
KT-07 & 6 & 2.15 & Compliance \\
\hline
\end{tabular}

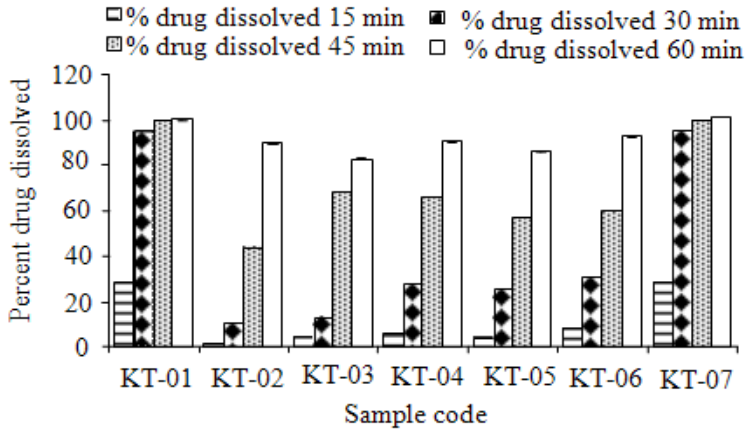

Fig. 2: Dissolution pattern of ketoprofen tablets in buffer stage (KT-1 to KT-7)

From Table 1 it was observed that 2 brands (KT-03 and KT-07) did not comply with BP specification in acid stage since small crack was observed in these brands. However, at the buffer stage all brands were found to comply with BP specification as illustrated in Fig. 1.

Dissolution study: The dissolution study of ketoprofen tablet was carried out in both acid and buffer stages. All the brands satisfied with USP specification in both stages. Result found in the dissolution study of ketoprofen tablet in acid stage was presented in Table 2. Also in the buffer stage of dissolution study all Ketoprofen tablet brands were found to be complied with USP specification (Fig. 2).

Dissolution study of four ketoprofen capsule brands was carried out only in buffer stage. The result was shown in Fig. 3. All the four brands showed excellent dissolution rate and were found to be dissolved $>80 \%$ of the labeled amount after $45 \mathrm{~min}$ which satisfied USP specification (Fig. 3).

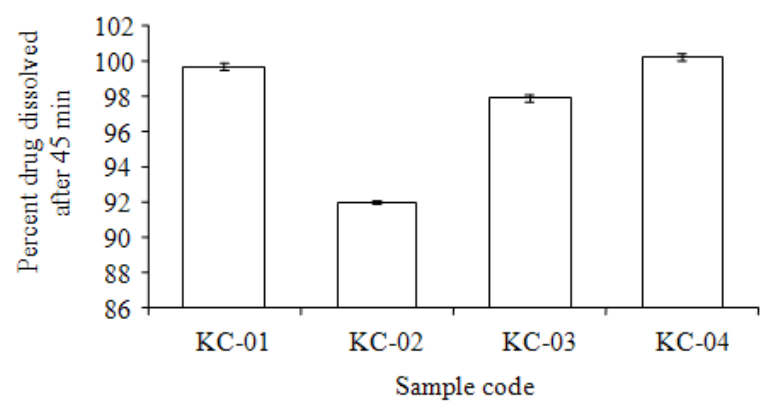

Fig. 3: Dissolution pattern of ketoprofen SR capsules in buffer stage (KC-1 to $\mathrm{KC}-4)$

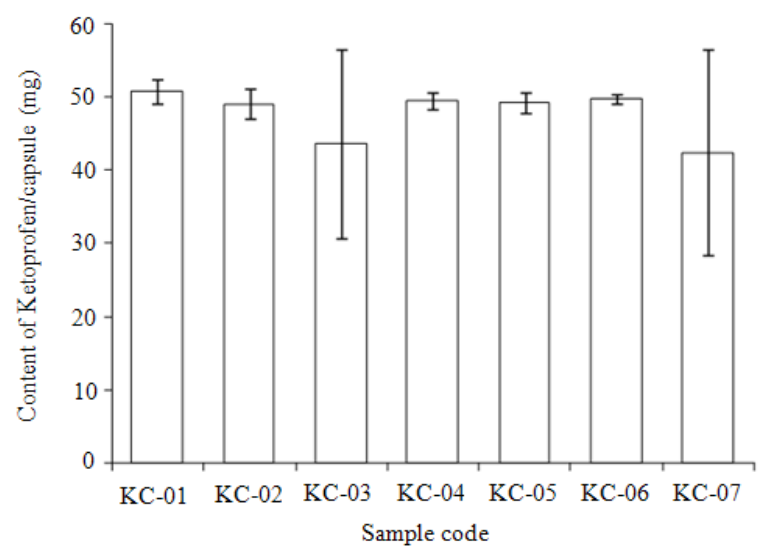

Fig. 4: Potency of different brands of ketoprofen tablets (claimed contents of each tablet is $50 \mathrm{mg}$ )

Potency determination: The potency of ketoprofen tablet and capsule brands was determined by UV spectroscopic method and the amount of ketoprofen present in the sampled 7 tablet brands was shown in Fig. 4 . 


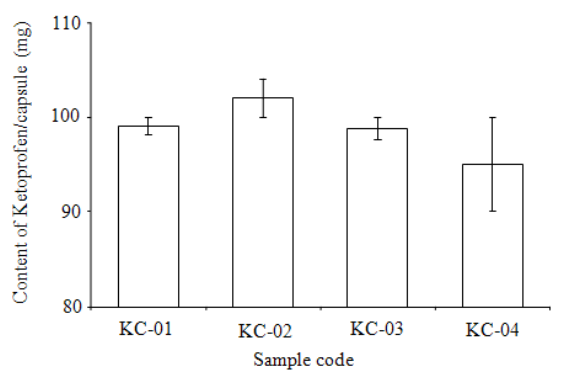

Fig. 5: Potency of different brands of ketoprofen capsules ((claimed contents of each capsule is $100 \mathrm{mg})$ )

We found that among tablet preparations only 2 brands (KT-3 and KT-7) were found to be noncompliant with BP specification. These two brands contained less active ingredient and may not be able to produce the effect that is naturally expected after administration of a standard drug.

The potency of 4 brands of ketoprofen capsule was also determined by UV spectroscopic method and all brands complied with BP specification (Fig. 5).

\section{DISCUSSION}

All marketed ketoprofen capsule brands taken in this study were found to demonstrate satisfactory pattern of disintegration, in respect of the specification of official compendia. Among tablet brands only KT-03 and KT-07 were found non-compliant at the in-vitro acid stage of disintegration. Small cracks were found in these two brands. This could be due to some minor manufacturing defects associated with these particular brands. In fact finding an optimized manufacturing procedure to design a best compromised tablet is always challenging (Lachman et al., 1986) and these two brands are not among the correctly compromised products.

Despite the noncompliance with acid stage of disintegration two tablet brands (KT-03 and KT-07) complied with the official specification at buffer stage of disintegration as well as in dissolution study. This is not contadictory since none should expect a linear relation among dissolution and disintegration. However the rationale to include disintegration test for studying release pattern is that as the tablet breaks down into small particles it offers greater surface area for dissolving media (Lachman et al., 1986) as shown by two tablet brands, KT-03 and KT-07. They broke down in the buffer stage (where it complied with BP specification) and were found to pass the dissolution test as per expectation.

In respect of potency, except two tablet brands (KT-03 and KT-07) all other ketoprofen tablet and capsule brands showed excellent result. These were potent enough to meet recommended official compendia. The lower potency of two tablet brands may be due to the degradation of active ingredient at the time of manufacturing or shelf-life.

\section{CONCLUSION}

The study covered only some selected brands of ketoprofen solid dosage products subject to the availability in local market. These are not sufficient to show the overall quality of this family of drugs. However, this study will provide a basis for in-vivo bioavailability studies of these brands to draw conclusion regarding quality status of these samples.

\section{ACKNOWLEDGEMENT}

The researchers would like to thank Beximco Pharmaceuticals Ltd. Dhaka and Eskayef Bangladesh Limited for providing standard sample of ketoprofen sustained release pellets and ketoprofen powder respectively which facilitated the present work.

\section{REFERENCES}

Aboul-Enein, H.Y., A.G. Dal and M. Tuncel, 2003. A validated method development for ketoprofen by a flow-injection analysis with UVdetection and its application to pharmaceutical formulations. II Farmaco, 58: 419-422. DOI: 10.1016/S0014$827 \mathrm{X}(03) 00061-2$

Blanco, M., J. Coello, H. Iturriaga, S. Maspoch and S. Alaoui-Ismaili, 1997. UV-spectrophotometric determination of ketoprofen and parabenin a gel preparation by partial least-squares calibration. Fresenius' J. Anal. Chem., 357: 967-972. DOI: $10.1007 / \mathrm{s} 002160050283$

Chambliss, W.G., D.A. Chambliss., R.W. Cleary, A.B. Jones and E.C. Harland et al., 1984. Development and evaluation of enteric coated penicillamine tablets. J. Pharm. Sci., 73: 1215-1219. DOI: 10.1002/jps.2600730908

Lachman, A., H.A. Liberman and J.L. Kanig, 1986. The Theory and Practices of Industrial Pharmacy. 3rd Edn., Lea and Febiger, Philadelphia, USA., ISBN: 10: 0812109775, pp: 902.

Maheswari, R.K., S. Sharma, N. Rai and M.S. Rajput, 2010. Simple titrimetric method to estimate ketoprofen in bulk using mixed hydrotrophy. J. Pharm. Res., 3:442-443.

Murshid, G.M.M., D.R. Paul, M.G. Hossain, A.A. Rahman and M. Saifuzzaman et al., 2006. Quality assessment of Indomethacin capsules marketed by Bangladeshi Pharmaceutical Industries. Khulna Univ. Stud., 7: 83-86.

Parker, K., 2005. Goodman and Gilman's The Pharmacological Basis of Therapeutics. 11th Edn., McGraw-Hill Professional, New York, pp: 1984. 